\title{
The Kleene equality for graphs
}

\author{
Arnaud Carayol and Didier Caucal \\ IrISA, Campus de Beaulieu, 35042 Rennes Cedex, France, \\ \{Arnaud.Carayol, Didier. Caucal\}@irisa.fr
}

\begin{abstract}
In order to generalize the Kleene theorem from the free monoid to richer algebraic structures, we consider the non deterministic acceptance by a finite automaton of subsets of vertices of a graph. The subsets accepted in such a way are the equational subsets of vertices of the graph in the sense of Mezei and Wright. We introduce the notion of deterministic acceptance by finite automaton. A graph satisfies the Kleene equality if the two acceptance modes are equivalent, and in this case, the equational subsets form a Boolean algebra. We establish that the infinite grid and the transition graphs of deterministic pushdown automata satisfy the Kleene equality and we present families of graphs in which the free product of graphs preserves the Kleene equality.
\end{abstract}

\section{Introduction}

In the case of the free monoid, Kleene theorem states the equivalence between acceptance by non-deterministic finite state automata and acceptance by deterministic and complete automata. As a consequence the regular sets of words are closed under complementation and form a Boolean algebra. For arbitrary monoids, the regular subsets (which are the subsets of the monoid accepted by finite automata labeled by elements of this monoid) are closed under union but are not, in general, closed under complementation. However there are well-known examples of monoids for which the regular subsets form a Boolean algebra: the free commutative monoid [GS64], the trace monoid with transitive independence relations [Sak87], the context-free groups [Sén96]... As these monoids are not free, the acceptance by deterministic and complete automata no longer provides the closure under complementation.

The goal of this article is to explain the closure under complementation of the regular subsets of such monoids by a notion of deterministic acceptance by finite state automaton. In order to do so, we consider the acceptance by finite automata of subsets of vertices of a colored graph.

A finite automaton is simply a finite colored graph whose vertices are called states, together with a set of final states. The run of the automaton $A$ on a graph $G$ is a relation between the vertices of the graph $G$ and the states of $A$. It is the smallest relation $\rho$ such that if a vertex $x$ of $G$ and a state $q$ of $A$ are colored by a same color, then $(x, q)$ also belongs to $\rho$ and such that if $(x, p)$ belongs to $\rho$ and there is a $a$-labeled edge from $x$ to $y$ in $G$ and a $a$-labeled transition from $p$ to $q$ in $A$, then $(y, q)$ belongs to $\rho$. Intuitively, the colored vertices of the graph 
act as starting points for the automaton and the colored states in the automaton play the role of initial states. The subset of vertices accepted by $A$ is the set of vertices coupled by $\rho$ with at least one final state. We denote $\operatorname{AFS}(G)$ the set of all subsets of vertices accepted by a finite automaton running on $G$. The set $\operatorname{AFS}(G)$ is in fact the set of all equational subsets (in the sense of Mezei and Wright [MW67]) of the subset algebra associated to $G$.

This framework encompasses the case of the monoids previously mentioned when considering their Cayley graphs [Cay78]. Every monoid $\mathcal{M}$ finitely generated by a subset $P \subseteq M$ can be represented by its Cayley graph $\mathcal{C}(\mathcal{M}, P)$ whose vertices are the elements of the monoid and where an edge labeled by $p \in P$ represent the product on the right by $p$ and where the neutral element is colored by $\iota$. For instance, the Cayley graphs of the free monoids over two letters $\{a, b\}$ is the full binary tree labeled by $a$ and $b$ with its root colored by $\iota$. The subsets of the Cayley graph of $\mathcal{M}$ accepted by a finite automaton are the regular subsets of $\mathcal{M}$.

The notion of deterministic acceptance is fairly simple: the run of an automaton is deterministic and complete if it associates one and only one state to each vertex of the graph. The set of all subsets of vertices accepted by $A$ with a deterministic and complete run is denoted $\operatorname{DAFS}(G)$. A graph $G$ is said to satisfy the Kleene equality if $\operatorname{AFS}(G)=\operatorname{DAFS}(G)$. In this case, $\operatorname{AFS}(G)$ is a Boolean algebra.

To substantiate the pertinence of the notion of deterministic acceptance, we show that the Cayley graph of the free commutative monoid with two generators, the rooted graphs of deterministic pushdown automata [MS85] (which contain the Cayley graphs of context-free groups) and the rooted deterministic prefix-recognizable graphs [Cau96] all satisfy the Kleene equality. Finally we provide sufficient conditions for the free product of graphs to preserve the Kleene equality.

\section{Preliminaries}

The inverse of a relation $R \subseteq P \times P$ is $R^{-1}:=\{(q, p) \mid(p, q) \in R\}$. The image of $Q \subseteq P$ by $R$ is $R(Q):=\{p \in P \mid \exists q \in Q,(q, p) \in R\}$. The product of two relations $R$ and $S$ is $R \cdot S:=\{(p, r) \mid \exists q \in P,(p, q) \in R$ and $(q, r) \in S\}$.

Colored graphs A colored graph $G$ labeled by a finite set $\Sigma$ and colored by a finite set $C$ is a subset of $(V \times \Sigma \times V) \cup(C \times V)$ for some countable $V$. The set of vertices of $G$ is $V_{G}:=\{u \in V \mid \exists v \in V, a \in \Sigma, c \in C,(u, a, v) \in G$ or $(v, a, u) \in$ $G$ or $(c, u) \in G\}$, its set $\Sigma_{G}$ of labels is $\left\{a \in \Sigma \mid \exists u, v \in V_{G},(u, a, v) \in G\right\}$ and its set $C_{G}$ of colors $\left\{c \in C \mid \exists u \in V_{G},(c, u) \in G\right\}$. If $(u, a, v)$ belongs to $G$, we will say that there is an $a$-labeled edge from $u$ to $v$. If $(c, u)$ belongs to $G$, we say that $u$ is colored by $c$.

A graph $H$ is a subgraph of $G$ if $H \subseteq G$ and it is a covering subgraph if $V_{H}=V_{G}$.

A path in $G$ is a sequence $u_{0} a_{1} u_{1} \ldots a_{n} u_{n} \in V_{G}\left(\Sigma_{G} V_{G}\right)^{*}$ such that for all $i \in[1, n-1],\left(u_{i}, a_{i+1}, u_{i+1}\right) \in G$. A graph $G$ is accessible from its colors if for all $x \in V_{G}$, there exists a path from some colored vertex $i \in V_{G}$ to $x$. 
A graph $G$ is rooted if there exists a color $c$ and a vertex $r$ called the root colored by $c$ such that $r$ is the only vertex colored by $c$ and every vertex is reachable from $r$.

A graph $G$ is deterministic if for all $(u, a, v) \in G$, if $\left(u, a, v^{\prime}\right) \in G$ then $v=v^{\prime}$ and for all $(c, u) \in G$, if $\left(c, u^{\prime}\right) \in G$ then $u=u^{\prime}$. A graph $G$ is (source) complete if for all $u \in V_{G}$ and $a \in \Sigma$, there exists $v \in V_{G}$ such that $(u, a, v) \in G$.

A morphism $\varphi$ from a graph $G$ to a graph $H$ is a mapping from $V_{G}$ to $V_{H}$ such that $(u, a, v) \in G$ implies $(\varphi(u), a, \varphi(v)) \in H$ and $(c, u) \in G$ implies $(c, \varphi(u)) \in H$.

For every graph $G$ with $C_{G} \cap \Sigma_{G}=\emptyset$, we define the subset algebra of $G$ which is a unary algebra over the signature $C_{G} \cup \Sigma_{G}$ where the symbols in $C_{G}$ are constants and the symbols in $\Sigma_{G}$ are unary functions. Its carrier is $2^{V_{G}}$ and symbols in $C_{G} \cup \Sigma_{G}$ are interpreted in the following way: for all $c \in C_{G}$, $\mathbf{c}=\left\{v \in V_{G} \mid(c, v) \in G\right\}$ and for all $a \in \Sigma$ and $M \subseteq V_{G}, \mathbf{a}(M)=\{v \mid \exists u \in$ $M,(u, a, v) \in G\}$.

Monoids and their Cayley graphs. A monoid $\mathcal{M}=(M, \cdot)$ is given by a set $M$ and an associative product · admitting a neutral element $1_{M} \in M$. The product is extended to subsets of $M$ by taking for all $P, Q \subseteq M, P \cdot Q:=$ $\{p \cdot q \mid p \in P$ and $q \in Q\}$. For all $P \subseteq M, P^{*}=\bigcup_{i \in \mathbb{N}} P^{i}$ where $P^{0}=\left\{1_{M}\right\}$ and $P^{i+1}=P^{i} \cdot P$. The set of all regular subsets of $\mathcal{M}$ denoted by $\operatorname{Reg}(\mathcal{M})$ is the smallest set containing the finite sets and closed under union, concatenation and the star operation.

A monoid $\mathcal{M}=(M, \cdot)$ is finitely generated by a finite subset $P$ of $M$ if $M=P^{*}$. Its Cayley graph $\mathcal{C}(M, P)$ is labeled by $P$ and colored by $\iota$ and defined by:

$$
C(M, P):=\left\{(m, p, m \cdot p \mid m \in M \text { and } p \in P\} \cup\left\{\left(\iota, 1_{M}\right)\right\} .\right.
$$

\section{Non-deterministic and deterministic finite state acceptance}

In Subsection 3.1, we present the equational subsets of vertices of a graph as the subsets accepted by finite state automata running on the graph. In Subsection 3.2 , we introduce the notion of subsets of vertices of a graph deterministically accepted by finite automata and state the generalization of the Kleene equality. Finally in Subsection 3.3, we compare this new notion with the well-known notion of recognizable subsets [MW67].

\subsection{Non-deterministic finite state acceptance}

A finite automaton $A$ is a finite colored graph whose vertices are called states together with a finite set of final states $F \subseteq V_{A}$.

The run of a finite automaton $A$ on a graph $G$ is the smallest relation $\rho \subseteq$ $V_{G} \times V_{A}$ satisfying:

- for all $c \in C_{G} \cap C_{A}$, all $x \in V_{G}$ and $p \in V_{A}$, if $(c, x) \in G$ and $(c, p) \in A$ then $(x, p) \in \rho$ 
- for all $a \in \Sigma_{G} \cap \Sigma_{A}, x, y \in V_{G}$ and $p, q \in V_{A}$, if $(x, p) \in \rho,(x, a, y) \in G$ and $(p, a, q) \in A$ then $(y, q) \in \rho$.

The subset of $V_{G}$ accepted by $A$ is $\rho^{-1}(F)$. The subsets of $G$ accepted by some finite automaton will be called the subsets accepted by finite state (AFS for short) and will be designated by $\operatorname{AFS}(G)$.

Intuitively, the colored vertices of the graph play the role of starting point for the automaton. We say that the automaton goes through an $a$-labeled edge $(x, a, y) \in G$ if for some edge $(p, a, q) \in A$, we have $(x, p) \in \rho$ and $(y, q) \in \rho$.

These subsets are the equational subsets (as originally defined in [MW67]) of the subset algebra of $G$. In fact for all $q \in V_{A}$, the sets $\rho^{-1}(\{q\})$ are the smallest solution (for the inclusion) of the following finite set of equations on the subset algebra associated to $G$ :

$$
X_{q}=\bigcup_{(c, q) \in A} \mathbf{c} \quad \cup \bigcup_{(p, a, q) \in A} \mathbf{a}\left(X_{p}\right) .
$$

In [Cou89], a characterization of equational subsets by finite automata is provided. Our definition differs slightly in the definition of the run on the automaton.

For all finite automata $A$ and $B$ with $V_{A} \cap V_{B}=\emptyset$, the run of $A \cup B$ on $G$ is the union of the run of $A$ on $G$ and of the run of $B$ on $G$. Hence $A F S(G)$ is closed under union.

Proposition 1. For all $G, \operatorname{AFS}(G)$ is closed under union and contains $\emptyset$.

It is well-known that $\operatorname{AFS}(G)$ is not in general closed under complementation. In fact, remark that $V_{G}$ does not, in general, belongs to $\operatorname{AFS}(G)$ : if a vertex $x$ of $G$ is not accessible from any colored vertex, then it does not belong to any set in $\operatorname{AFS}(G)$. As we investigate cases for which $\operatorname{AFS}(G)$ is a Boolean algebra, it is reasonable to assume that the graphs under consideration are accessible from their colors. In the following, we will always assume that the graphs under consideration are accessible from their colors.

Example 1. Consider the graph Stacks associated to pushdown stacks over the alphabet $\Gamma=\{a, b\}$ :

$$
\begin{aligned}
& \text { Stacks }:=\left\{(u, x, u x) \mid x \in\{a, b\} \text { and } u \in \Gamma^{*}\right\} \\
& \cup\left\{(u x, \bar{x}, u) \mid x \in\{a, b\} \text { and } u \in \Gamma^{*}\right\} \cup\{(\iota, \varepsilon)\} .
\end{aligned}
$$

The vertices are the stacks over $\Gamma$ (which are simply words in $\Gamma^{*}$ ) and the edges represent the basic operations on stacks: an edge labeled by $a$ (resp. $b$ ) represents the push of the letter $a$ (resp. $b$ ) on top of the stack and an edge labeled by $\bar{a}$ (resp. $\bar{b}$ ) the removal of the top most letter of the stack if it is an $a$ (resp. $b$ ). The empty stack is colored by $\iota$.

Every set $V \in \operatorname{AFS}$ (Stacks) can be seen as the set of stack contents appearing in a final reachable configurations of some pushdown automaton. In fact if we 
omit the input alphabet, a pushdown automaton is simply a finite automaton labeled by the operations $\{a, b, \bar{a}, \bar{b}\}$. In [Büc64], Büchi showed that these sets are regular. Hence AFS(Stacks) $=\operatorname{Reg}\left(\Gamma^{*}\right)$ is a Boolean algebra.

Figure 1 presents the run $\rho$ of a finite automaton $A$ on Stacks. The set of states of $A$ associated by $\rho$ to a vertex $x$ of Stacks are written in boldface next to $x$. If we take $\left\{r_{1}, l_{1}\right\}$ as set of final states for $A, A$ accepts the set $\{a a, b b\}^{*} \cdot\{a, b\}$.

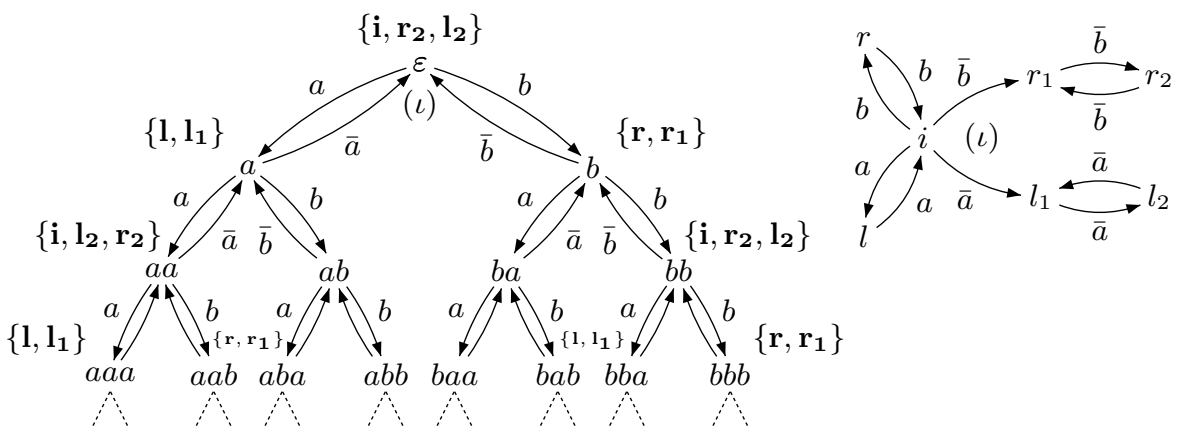

Fig. 1. The run (on the left) on Stacks of a finite automaton $A$ (on the right).

Other meaningful examples of graphs for which $\operatorname{AFS}(G)$ is a Boolean algebra are provided by considering the Cayley graphs of finitely generated monoids. The sets accepted by finite automata running on the Cayley graph of a finitely generated monoid are the regular subsets of this monoid.

Proposition 2. For any monoid $\mathcal{M}$ finitely generated by $P$,

$$
\operatorname{AFS}(\mathcal{C}(\mathcal{M}, P))=\operatorname{Reg}(\mathcal{M}) .
$$

Example 2. A first simple example of Cayley graph is the Cayley graph $\Delta_{2}$ of the free monoid $\{a, b\}^{*}$ presented in Figure 2. Consider now the free commutative monoid with two generators $\left(\mathbb{N}^{2},+\right)$ where + designates the componentwise addition. Its Cayley graph Grid with respect to the generating set $\{(0,1),(1,0)\}$ is the infinite grid depicted in Figure 2. By Proposition 2, the subsets accepted by finite automata on Grid are the regular subsets of $\left(\mathbb{N}^{2},+\right)$ which are also known as the semi-linear sets of $\mathbb{N}^{2}$. From [GS64], we know that AFS(Grid) is a Boolean algebra.

By a standard powerset construction, a non deterministic automaton $A$ can be transformed into a deterministic and complete automaton accepting the same subset.

Proposition 3 ([MW67]). For all graph $G$, every set in $\operatorname{AFS}(G)$ is accepted by a deterministic and complete automaton.

As shown in Example 1, a deterministic automaton does not necessarily have a deterministic behavior: its run can assign several states to the same vertex. We will say that such a run is non deterministic. 

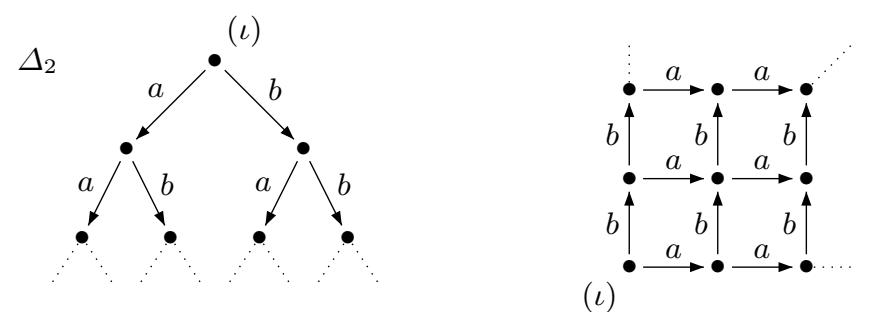

Grid

Fig. 2. The Cayley graph $\Delta_{2}$ of the free monoid $\{a, b\}^{*}$ and the Cayley graph Grid of $\left(\mathbb{N}^{2},+\right)$ with $a=(1,0)$ and $b=(0,1)$.

\subsection{Deterministic finite state acceptance}

We lift the notion determinism and completeness from the automaton to the behaviour of the automaton : its run.

Definition 1. The run $\rho$ of a finite automaton $A$ on a graph $G$ is said to be deterministic and complete if for every vertex $x \in V_{G}$, there exists one and only one state $p \in V_{A}$ such that $(x, p) \in \rho$.

In other terms, $\rho$ is a mapping. In this case, we will adopt the functional notation and write $\rho(x)=p$ instead of $(x, p) \in \rho$.

The set of all subsets of $G$ accepted by a finite automaton having a deterministic and complete run on $G$ is written $\operatorname{DAFS}(G)$. In the following we will simply say that these subsets of vertices are deterministically accepted. By definition, for all graph $G, \operatorname{DAFS}(G)$ is included in $\operatorname{AFS}(G)$.

Note that every set $V \in \operatorname{DAFS}(G)$ is deterministically accepted by a deterministic automaton. However note that contrary to what we obtained for $\operatorname{AFS}(G)$, we can no longer assume that this automaton is complete (see Subsection 3.3 for a discussion on this fact).

As the automaton is not necessarily complete, its run on a graph $G$ induces a subgraph of $G$ obtained by only keeping the edges of $G$ borrowed by the automaton. For any graph $G$ and any finite automaton $A$ with a deterministic run $\rho$ on $G$, the graph of the run $\rho$ is the graph $G_{\rho}$ defined by: $G_{\rho}:=\{(u, a, v) \in$ $G \mid(\rho(u), a, \rho(v)) \in A\}$. As $\rho$ is complete, $G_{\rho}$ is a covering subgraph of $G$.

Example 3. Consider the finite automaton $A$ of Figure 3. Its run on Grid (cf.

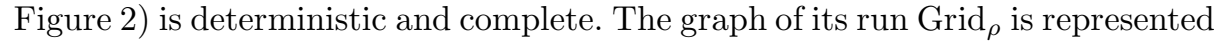
in Figure 3. If we take $\{r, s\}$ as set of final states for $A, A$ accepts the diagonal of the grid $\{(n, n) \mid n \in \mathbb{N}\}$.

For any set $V \in \operatorname{DAFS}(G)$, there exists a finite automaton $A$ with a set $F \subseteq V_{A}$ of final states and a deterministic run $\rho$ on $G$ such that $V=\rho^{-1}(F)$. As $\rho$ is a mapping, $V_{G} \backslash V=\rho^{-1}\left(V_{A} \backslash V_{F}\right)$ and it follows that $\operatorname{DAFS}(G)$ is closed under complementation.

Proposition 4. For all graph $G$, $\operatorname{DAFS}(G)$ is closed under complementation. 

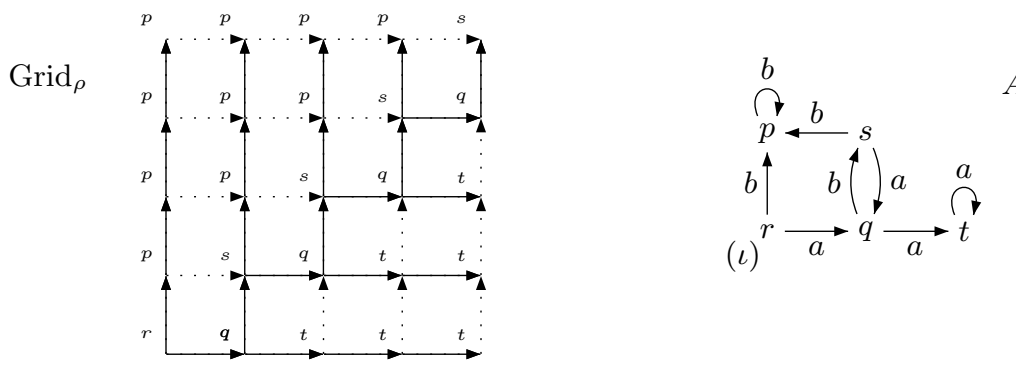

Fig. 3. A finite automaton $A$ with a deterministic run $\rho$ on Grid and the graph of its run $\operatorname{Grid}_{\rho}$.

However DAFS $(G)$ is not in general closed under union and intersection. For example consider the graph $G$ and the two automata $A$ and $B$ presented in Figure 4 . These two automata have a deterministic run on $G$ and by taking $f$ as unique final state, they accept $\{p\}$ and $\{s\}$ respectively. However $\{p, s\}$ does not belong to $\operatorname{DAFS}(G)$. In fact for all deterministic and complete run $\rho$, we have either $\rho(q)=\rho(p)$ or $\rho(q)=\rho(s)$.
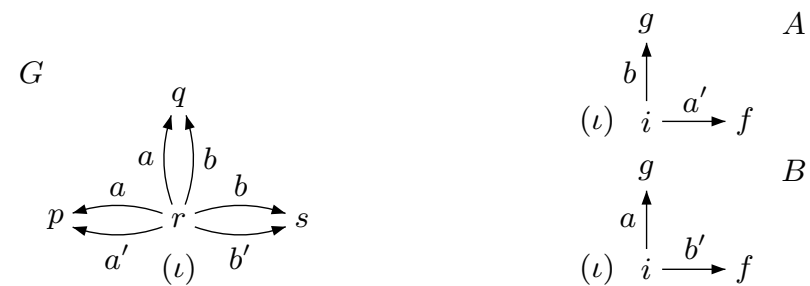

Fig. 4. Example of graph $G$ for which $\operatorname{DAFS}(G)$ is not closed under union.

If non deterministic and deterministic acceptances are equivalent on $G$, we say that $G$ satisfies the Kleene equality.

Definition 2. A graph $G$ satisfies the Kleene equality if $\operatorname{AFS}(G)=\operatorname{DAFS}(G)$.

As by Proposition $1, \operatorname{AFS}(G)$ is closed under union and contains the empty set and by Proposition 4, $\operatorname{DAFS}(G)$ is closed under complementation, it follows that $\operatorname{AFS}(G)$ is a Boolean algebra.

Proposition 5. For any $G$, if $\operatorname{AFS}(G)=\operatorname{DAFS}(G)$ then $\operatorname{AFS}(G)$ is a Boolean algebra.

The Kleene equality states the equivalence between non-deterministic and deterministic acceptance by finite automata. A stronger requirement is that every non-deterministic run can be determinized. 
Definition 3. For any graph $G, G$ satisfies the strong Kleene equality if for every finite automaton $A$ with a run $\rho_{A}$ on $G$, there exists a finite automaton $B$ with a deterministic and complete run $\rho_{B}$ on $G$ and a relation $\eta \subseteq V_{B} \times V_{A}$ such that $\rho_{A}=\rho_{B} \cdot \eta$.

Intuitively, this means that for any finite automaton $A$ there exists an automaton $B$ with a deterministic run of $G$ such that the set of states associated to a vertex $x$ of $G$ is entirely characterized by the unique state $\rho_{B}(x)$ associated by $B$ to $x: \rho_{A}(x)=\eta\left(\rho_{B}(x)\right)$.

In particular, if $F_{A}$ is the set of final states of $A$, by taking $F_{B}=\eta^{-1}\left(F_{A}\right)$ as set of final states for $B, B$ accepts the same subset as $A$. Hence, if a graph satisfies the strong Kleene equality, it satisfies the Kleene equality.

Example 4. Consider the graph Stacks and the finite automaton $A$ presented in Example 1. The automaton $B$ presented in Figure 5 has a deterministic and complete run $\rho_{B}$ on Stacks and accepts the same language as $A$ if we take $\{p, s\}$ as final states. Moreover taking $\eta=\left\{(r, i),\left(r, r_{2}\right),\left(r, l_{2}\right),(s, l),\left(s, l_{1}\right),(p, r),\left(p, r_{1}\right)\right\}$, we have $\rho_{A}=\rho_{B} \cdot \eta$. In fact as Stacks is a rooted and deterministic pushdown transition graph, we will prove in Subsection 4.2 that it satisfies the strong Kleene equality.

( $\iota)$

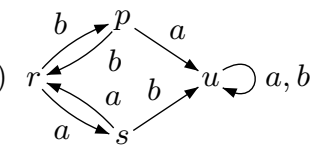

Fig. 5. An automaton $B$ with a deterministic and complete run on Stacks (cf. Ex. 1).

\subsection{Comparison with recognizable subsets}

The notion of recognizable subsets of a monoid was introduced by Eilenberg. Mezei and Wright extended this notion to algebras in [MW67]. For a graph $G$, a subset of vertices $V \subseteq V_{G}$ is recognizable if there exists a deterministic and complete automaton $A$ with $\Sigma_{A}=\Sigma_{G}$ and $C_{A}=C_{G}$ and a morphism $\varphi$ from $G$ to $A$ such that $V=\varphi^{-1}(\varphi(V))$. We write $\operatorname{Rec}(G)$ the set of all recognizable subsets of vertices of $G$.

It is well-known that for any graph $G, \operatorname{Rec}(G)$ is a Boolean algebra. In our setting, this notion can be captured by considering deterministic and complete automaton having a deterministic run.

Proposition 6. For all graph $G$, a subset $V$ of $V_{G}$ is recognizable if and only if it is deterministically accepted by a deterministic and complete finite automaton $A$ with $\Sigma_{A}=\Sigma_{G}$ and $C_{A}=C_{G}$. 
A direct consequence of this characterization is that for all graph $G$, we have $\operatorname{Rec}(G) \subseteq \operatorname{DAFS}(G) \subseteq \operatorname{AFS}(G)$. In the case of the free monoid $\{a, b\}^{*}$ (whose Cayley graph is $\Delta_{2}$ presented in Figure 2), it is well-known that $\operatorname{Rec}\left(\Delta_{2}\right)=$ $\operatorname{AFS}\left(\Delta_{2}\right)$. Hence $\Delta_{2}$ satisfies the Kleene equality. In general, $\operatorname{Rec}(G)$ is strictly included in $\operatorname{DAFS}(G)$. Consider for example the graph Grid presented in Figure 2: we have seen in Example 3 that $\{(n, n) \mid n \in \mathbb{N}\}$ belongs to DAFS(Grid) but does not belong to Rec(Grid). In fact, the recognizable subsets of Grid are finite unions of products of subsets in $\operatorname{Reg}((\mathbb{N},+))$.

The following proposition is well-known in the case of $\operatorname{AFS}(G)$.

Proposition 7. For all $G$, if $P \in \operatorname{Rec}(G)$ and $Q \in \operatorname{DAFS}(G)$ then $P \cap Q \in$ $\operatorname{DAFS}(G)$.

\section{Motivating examples}

\subsection{The grid}

In this section, we consider the Cayley graph Grid (presented in Example 2) of the free commutative monoid with two generators. In [ES69], the authors establish that the regular subsets of this monoid are unambiguous. A regular subset $R$ is unambiguous if it is accepted by a finite automaton $A$ with a finite set of initial states and such that for all $r \in R$, there exists exactly one computation of $A$ accepting $r$. Note that the run on the Cayley graph of the monoid of an unambiguous automaton is, in general, neither deterministic (as several initial states are allowed) nor complete (as the definition does not imply the existence of a path reaching the elements that do not belong to $R$ ). In fact, the unambiguous regular subsets are in general not closed under complementation.

The proof that Grid satisfies the strong Kleene equality is quite involved and starts from the unambiguous characterization of [ES69].

Theorem 1. The Cayley graph Grid of the free commutative monoid with two generators satisfies the strong Kleene equality.

We conjecture that this result extends to the Cayley graphs of freely generated monoids with an arbitrary number of generators.

\subsection{Graphs of deterministic pushdown automata}

In [Sén96], the author proves that the regular subsets of a context-free group form a Boolean algebra. In [MS85], Muller and Schupp proved that Cayley graphs of context-free groups are rooted deterministic pushdown transition graphs. We establish that all rooted deterministic pushdown transition graphs satisfy the strong Kleene equality. In particular, it follows that the Cayley graphs of the context-free groups satisfy the strong Kleene equality.

Recall that a (real-time) pushdown automaton is a finite set $R$ of rules of the form $(p A, a, q U)$ with $p, q \in Q, A \in P, U \in P^{*}, a \in \Sigma$, where $Q, P, \Sigma$ are 
disjoint alphabets of respectively states, pushdown letters and labels. A configuration of the pushdown graph is a word $q w$ where $q \in Q$ and $w \in P^{*}$. The transition graph $P(R)$ of any pushdown automaton $R$ is the uncolored graph $P(R):=\left\{(u w, a, v w) \mid(u, a, v) \in R \wedge w \in P^{*}\right\}$. The rooted transition graph $P(R, r)$ of $R$ from any configuration $r \in Q P^{*}$ is the graph $P(R, r)$ obtained from $P(R)$ by coloring the vertex $r$ by a color $\iota$ and by restricting to the vertices accessible from $r$. Figure 6 illustrates this notion.

$$
\begin{array}{ll}
(p A, a, P A A) & Q=\{p, q\} \\
(p A, b, q) & P=\{A, B\} \\
(q A, c, q) &
\end{array}
$$

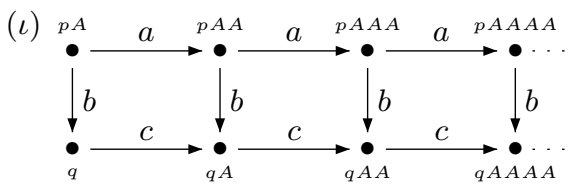

Fig. 6. A pushdown transition graph rooted in $p A$.

Theorem 2. Every rooted deterministic pushdown transition graph $G$ satisfies the strong Kleene equality.

This result extends to the rooted deterministic prefix-recognizable graphs introduced in [Cau96]. A prefix-recognizable relation on words over a finite alphabet $\Gamma$ is a finite union of relations of the form $(U \times V) \cdot W$ where $U, V$ and $W$ belong to $\operatorname{Reg}\left(\Gamma^{*}\right)$. A prefix-recognizable graph labeled by $\Sigma$ is an uncolored graph defined by a family $\left(R_{a}\right)_{a \in \Sigma}$ of prefix-recognizable relations on words over $\Gamma^{*}$ and is equal to $\left\{(u, a, v) \mid u, v \in \Gamma^{*}, a \in \Sigma\right.$ and $\left.(u, v) \in R_{a}\right\}$. For any prefix-recognizable graph $G$, the prefix-recognizable graph $G / r$ rooted in $r \in V_{G}$ is the graph obtained by restricting $G$ to the set of vertices accessible from $r$ and adding the color $\iota$ on $r$. Figure 7 presents a rooted deterministic prefix-recognizable graph which is not a rooted pushdown graph.

$$
\begin{aligned}
& \Sigma=\{a, b\} \\
& \Gamma=\{c\} \\
& R_{a}=(\{\varepsilon\},\{c\}) \cdot c^{*} \\
& R_{b}=\left(c^{+},\{\varepsilon\}\right) \cdot c^{*}
\end{aligned}
$$

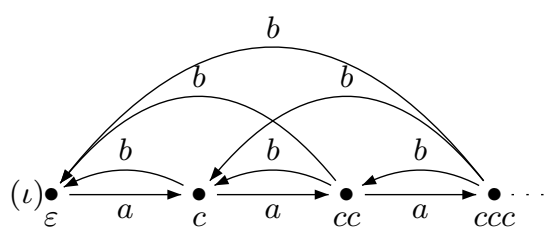

Fig. 7. A prefix-recognizable graph rooted in $\varepsilon$.

Theorem 3. Every rooted deterministic prefix-recognizable graph satisfies the strong Kleene equality. 


\subsection{Free product of rooted graphs}

In order to obtain more graphs satisfying the Kleene equality, we consider the free product of rooted graphs. In [Sak87], the author established that for two disjoint monoids $\mathcal{M}$ and $\mathcal{N}$ such that $\operatorname{Reg}(\mathcal{M})$ and $\operatorname{Reg}(\mathcal{N})$ are Boolean algebras then the regular subsets of the free product of $\mathcal{M}$ and $\mathcal{N}$ form a Boolean algebra. We naturally extend the free product of two monoids to rooted graphs and show that on Cayley graphs, the free product preserves the strong Kleene equality.

Let $G$ and $H$ be two rooted graphs with respective roots $r_{G}$ colored by $g$ and $r_{H}$ colored by $h$ such that $V_{G} \cap V_{H}=\Sigma_{G} \cap \Sigma_{H}=\emptyset$ and $C_{G}=\{g\}$ and $C_{H}=\{h\}$. We take $V_{G}^{\prime}=V_{G} \backslash\left\{r_{G}\right\}$ and $V_{H}^{\prime}=V_{H} \backslash\left\{r_{H}\right\}$.

The set of vertices of the free product $G \otimes H$ of $G$ and $H$ is $S_{G} \cup S_{H}$ where $S_{G}=\left(V_{G}^{\prime} V_{H}^{\prime}\right)^{*} V_{G}^{\prime} \cup\left(V_{H}^{\prime} V_{G}^{\prime}\right)^{*}$ and $S_{H}=\left(V_{H}^{\prime} V_{G}^{\prime}\right)^{*} V_{H}^{\prime} \cup\left(V_{G}^{\prime} V_{H}^{\prime}\right)^{*}$. The graph $G \otimes H$ is rooted at empty sequence $\varepsilon$ and defined by:

$$
G \otimes H:=\bigcup_{u \in S_{G}} u \cdot[H] \cup \bigcup_{u \in S_{H}} u \cdot[G] \cup\{(\iota, \varepsilon)\}
$$

where $[G]$ (resp. $[H]$ ) designates the graph obtained by renaming the root $r_{G}$ (resp. $r_{H}$ ) by the empty sequence and for all graph $u \cdot[G]($ resp. $u \cdot[H])$ is the graph $\{(u v, a, u w) \mid(v, a, w) \in G\}$ (resp. $\{(u v, a, u w) \mid(v, a, w) \in H\})$.

In particular, the free product of the Cayley graphs of two disjoint monoids is the Cayley graph of the free product of these two monoids.

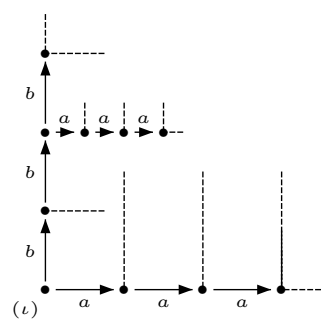

Fig. 8. The free product of two semi-lines labeled by $a$ and by $b$ respectively.

A first simple case in which the free product preserves the strong Kleene equality is when one of the two graphs has no incoming edge to its root.

Proposition 8. If $G$ has no incoming edge to its root and if $G$ and $H$ both satisfy the Kleene equality then their free product $G \otimes H$ also satisfies the Kleene equality.

In particular, it follows that the free-product of Grid and of any deterministic rooted pushdown graph satisfy the strong Kleene equality.

Theorem 4. For any two disjoint monoids $\mathcal{M}$ and $\mathcal{N}$ finitely generated by $P \subset$ $M$ and $Q \subset N$, if the $\mathcal{C}(\mathcal{M}, P)$ and $\mathcal{C}(\mathcal{N}, Q)$ both satisfy the strong Kleene equality then $\mathcal{C}(\mathcal{M}, P) \otimes \mathcal{C}(\mathcal{N}, Q)$ also satisfies the strong Kleene equality. 
The proof of Theorem 4 is an adaptation of Theorem 5.2 of [Sak87].

Example 5. Consider the free partially commutative monoid with four generators $a, b, c, d$ satisfying the equations $a b=b a$ and $c d=d c$. Its Cayley graph is the free product of two grids respectively labeled by $\{a, b\}$ and $\{c, d\}$. By Theorem 4 and Theorem 1, its Cayley graph satisfies the strong Kleene equality.

\section{Conclusion}

In this article, we introduced the natural notion of deterministic acceptance by finite automata on colored graphs. We showed that it allows one to extend the Kleene theorem on free monoid to richer algebraic structures such as the free commutative monoid with two generators, the context-free groups and the trace monoid with a transitive independence relations with at most two independent generators (as their Cayley graphs are free products of grids and lines). We think that this notion brings new insight on the closure by complementation of the regular subsets of these monoids.

This work leaves several open questions. In particular, we conjecture that infinite grids of arbitrary dimension satisfy the strong Kleene equality. It remains to extend the notion of deterministic acceptance to capture the equational subsets of richer structures such as for example the canonical graphs associated to stacks of stacks [Car05]. Finally we can readily extend these notions to relational structures. However it remains to exhibit pertinent relational structures for which the equational subsets form a Boolean algebra.

\section{References}

[Büc64] J. Büchi. Regular canonical systems. Arch. Math. Logik Grundlag., 6:91-111, 1964.

[Car05] A. Carayol. Regular sets of higher-order pushdown stacks. In Proc. MFCS '05, pages 168-179, 2005.

[Cau96] D. Caucal. On infinite transition graphs having a decidable monadic theory. In Proc. ICALP 96, volume 1099 of LNCS, pages 194-205, 1996.

[Cay78] A. Cayley. On the theory of groups. Proc. London Math. Soc., 9:126-133, 1878.

[Cou89] B. Courcelle. On recognizable sets and tree automata. In Resolution of Equations in Algebraic Structures. Academic Press, 1989.

[ES69] S. Eilenberg and M. Schützenberger. Rational sets in commutative monoids. J. Algebra, 13:344-353, 1969.

[GS64] S. Ginsburg and E. Spanier. Bounded algol-like languages. Trans. Amer. Math. Soc., 113:333-368, 1964.

[MS85] D. Muller and P. Schupp. The theory of ends, pushdown automata, and secondorder logic. TCS, 37:51-75, 1985.

[MW67] J. Mezei and J. Wright. Algebraic automata and context free sets. Information and Control, 11:3-29, 1967.

[Sak87] J. Sakarovich. On regular trace languages. TCS, 52:59-75, 1987.

[Sén96] G. Sénizergues. On the rational subsets of the free group. Acta Informatica, 33:281-296, 1996. 